\title{
Cenários híbridos de aprendizagem e a configuração de comunidades virtuais no ensino superior
}

\author{
Hybrid learning scenarios and the design of virtual \\ communities in higher education
}

\section{Escenarios de aprendizaje híbrido y diseño de comunidades virtuales en la enseñanza superior}

\author{
JosÉ ANTÓNio MOREIRA* \\ JOANA CORREIA ${ }^{* *}$ \\ SARA DIAS-TRINDADE ${ }^{* * *}$
}

\begin{abstract}
A evolução das tecnologias digitais e das redes de comunicação propiciaram o surgimento de uma sociedade reticular marcada por mudanças acentuadas na economia e no mercado de trabalho, impulsionando o nascimento de novos paradigmas, modelos, processos de comunicação educacional e novos cenários de ensino e de aprendizagem. Esta realidade, exige, pois, que se pense, urgentemente, em criar e desenvolver estruturas que respondam a estas mudanças, com instituições digitalmente inovadoras, capazes de transformar a realidade educativa. Com efeito, integrar tecnologias audiovisuais em contexto educativo pode ser uma estratégia muito adequada para revitalizar a experiência educacional, e é precisamente, a criação de cenários híbridos de aprendizagem enriquecidos com a presença do audiovisual que pretendemos analisar, descrevendo o seu impacto na construção de uma comunidade virtual, a partir da análise qualitativa das perceções de vinte estudantes a frequentar o Ensino Superior, a nível de pós-graduação, tendo como referencial os modelos pedagógicos desenvolvido por Garrison e colaboradores (2000) e Moreira (2017). Os resultados revelam que a criação destes cenários híbridos, de um ecossistema de educação digital enriquecido com tecnologias audiovisuais, ancorado nos modelos referenciados, pode ter efeitos muito positivos na criação e desenvolvimento de comunidades virtuais de aprendizagem.
\end{abstract}

The evolution of digital technologies and communication networks has led to the emergence of a reticular society marked by significant changes in the economy and the labor market, driving the birth of new paradigms, models, educational communication processes, and new teaching and learning scenarios. This reality, therefore, demands urgent thought to create and develop structures that respond to these changes, with digitally innovative institutions capable of transforming the educational reality. Integrating audiovisual technologies in an educational context may be a very appropriate strategy to revitalize the educational experience, and it is precisely the creation of hybrid learning scenarios enriched with the presence of the audiovisual that we intend to analyze, describing its impact on the design of a virtual community. For that, we use the qualitative analysis of the perceptions of twenty students attending Higher Education, at postgraduate level, having as reference the pedagogical models developed by Garrison, Anderson \& Archer (2000) and Moreira (2017). The results reveal that the creation of these hybrid scenarios, of a digital education ecosystem enriched with audiovisual technologies, anchored in the referenced models, can have very positive effects on the creation and development of virtual learning communities.
Palavras-chave:

cenários de aprendizagem híbridos, modelo pedagógico virtual, comunidade virtual de aprendizagem, ensino superior

\section{Keywords:}

hybrid learning scenarios, virtual pedagogical model, virtual learning community, higher education 
La evolución de las tecnologías digitales y de las redes de comunicación ha propiciado el surgimiento de una sociedad reticular marcada por importantes cambios en la economía y el mercado laboral, que ha impulsado el nacimiento de nuevos paradigmas, modelos, procesos de comunicación educativa y escenarios de enseñanza y aprendizaje novedosos. Esta realidad exige una reflexión urgente para crear y desarrollar estructuras que respondan a estos cambios, con instituciones digitalmente innovadoras capaces de transformar la realidad educativa. Integrar las tecnologías audiovisuales en un contexto educativo puede ser una estrategia muy adecuada para revitalizar la experiencia educativa, y es la creación de escenarios híbridos de aprendizaje enriquecidos con la presencia del audiovisual lo que se pretende analizar en este artículo, y describir su impacto en el diseño de una comunidad virtual. Para ello, se utiliza el análisis cualitativo de las percepciones de veinte estudiantes que cursan estudios superiores, a nivel de posgrado, y se tiene como referencia los modelos pedagógicos desarrollados por Garrison y colaboradores (2000) y Moreira (2017). Los resultados revelan que la creación de estos escenarios híbridos, de un ecosistema educativo digital enriquecido con tecnologías audiovisuales, anclado en los modelos referenciados, puede tener efectos muy positivos en la creación de comunidades virtuales de aprendizaje.

\section{Palabras clave:}

escenarios de aprendizaje híbridos, modelo pedagógico virtual, comunidad de aprendizaje virtual, educación superior

Recebido: 15 de Julho de 2021 | Aceite para publicação: 17 de Novembro de 2021 |

Publicado: 3 de Janeiro de 2022

Como citar: Moreira, J. A., Correia, J. \& Dias-Trindade, S. (2022). Cenários híbridos de aprendizagem e a configuração de comunidades virtuais no ensino superior. Sinéctica, Revista Electrónica de Educación, (58), e1353. https://doi.org/10.31391/S2007-7033(2022)0058-002

\footnotetext{
* Doutor e Mestre em Ciências da Educação e Licenciado em História da Arte pela Universidade de Coimbra. Professor Associado com Agregação no Departamento de Educação e Ensino a Distância da Universidade Aberta. Diretor da Delegação Regional do Porto da Universidade Aberta. Linhas de investigação: educação digital, educação híbrida, ambientes virtuais de aprendizagem, competências digitais. E-mail: jmoreira@uab.pt/https://orcid.org/0000-00030147-0592

** Doutoranda em Sociologia, Iscte, Instituto Universitário de Lisboa. Assistente Convidada no Laboratório de Competências Transversais do Iscte, Instituto Universitário de Lisboa, Portugal. Centro de Investigação e Estudos de Sociologia (CIES-Iscte). Linhas de investigação: educação digital, educação híbrida, ambientes virtuais de aprendizagem, competências digitais. E-mail: Joana_Telma_Correia@iscte-iul.pt/http://orcid.org/0000-0002-0820-7562

*** Doutora em História. Universidade de Coimbra, Centro de Estudos Interdisciplinares, Faculdade de Letras (DHEEAA). Linhas de investigação: educação digital, educação híbrida, ambientes virtuais de aprendizagem, competências digitais. E-mail: sara.trindade@uc.pt/https://orcid.org/0000-0002-5927-3957
}

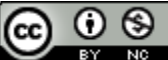




\section{INTRODUÇÃo}

$\mathrm{E}$

$\mathrm{m}$ tempos de profundas mudanças, de um mundo estruturado de uma forma complexa onde coabitam o analógico e o digital, o real e o virtual, o humano e a máquina, o offline e o online, do reconhecimento de que vivemos numa nova era marcada pela pandemia do COVID-19 e da vertiginosa evolução das tecnologias digitais, deparamo-nos com a necessidade de repensar modelos, processos de comunicação educacional e novos ecossistemas de aprendizagem. A crise de saúde pública e as experiências de ensino remoto de emergência online em toda a Europa e no mundo na primavera de 2020 têm permitido perceber que em vez de retornar totalmente à forma como as instituições de Ensino Superior funcionavam, é possível pensar numa educação mais híbrida, através de processos de inovação sustentada, que permitam combinar diferentes presenças, tempos, tecnologias e, sobretudo, articular diferentes espaços e ambientes de aprendizagem.

0 conceito do híbrido não pode ser entendido e reduzido apenas à combinação de ambientes físicos e virtuais de aprendizagem. Pelo contrário, o hibridismo na educação deve afirmar-se como um conceito de educação total caracterizado pelo uso de soluções combinadas, envolvendo a interação entre diferentes modelos, abordagens pedagógicas e recursos tecnológicos. A interação das abordagens pedagógicas é consequência direta e indireta da conjugação de diversos recursos, envolvendo espaços diferenciados. Essa interação implica a convivência de teorias muitas vezes consideradas mutuamente exclusivas, como é o caso da clássica oposição em torno das teorias behavioristas e das cognitivistas. A adoção de diferentes recursos tecnológicos é um dos caminhos, entre outros, para se efetivar o diálogo entre estas diferentes abordagens, uma vez que cada recurso implica, não apenas formas específicas de gerir o conhecimento, mas também, formas específicas de interação (Moreira \& Ferreira, 2021).

Ou seja, a educação híbrida deve ser entendida, por um lado, como uma estratégia dinâmica que envolve diferentes recursos tecnológicos, distintas abordagens pedagógicas e diferentes tempos e, por outro, como um processo de comunicação altamente complexo que promove uma série de interações entre atores humanos e não-humanos que podem ser bem sucedidas, desde que sejam incorporados todos estes elementos (Monteiro, Moreira \& Lencastre, 2015).

Nesta "nova" realidade híbrida, as salas de aula necessitam de estar conectadas a instrumentos e redes de conhecimento em permanente atualização, aproximando atores humanos e não-humanos. Esta centralidade da rede digital remete para variáveis comunicacionais significativas como a interação, ligação, conexão e participação, essenciais a um paradigma assente numa abordagem de Inteligência Humana Aumentada (Harasim, 2015), que promova e aumente a ação e interação humana e não que automatize ou reduza essa ação, como nas abordagens de Inteligência Artificial (IA).

Com efeito, a já referida vertiginosa evolução das tecnologias digitais, assim como a ampla disponibilidade de informações encontradas na web e o aparecimento do software livre têm incentivado a partilha entre indivíduos e a emergência espontânea de comunidades virtuais.

Em função disso, muitos investigadores têm procurado características dos sistemas biológicos para compreender, analisar e criar ecossistemas digitais, contudo 
esta tendência é relativamente recente e não há ainda uma definição consolidada, tão pouco existe um consenso relativamente à sua finalidade.

Cientistas que anteriormente representavam o mundo como máquinas, como mecanismos de regras lineares de causa e efeito, estão a mudar de metáfora, vendo os seus objetos de estudo, agora como ecossistemas digitais que são, na sua essência, análogos às comunidades ecológicas naturais, pois apresentam-se como sistemas complexos, dinâmicos e adaptativos e interagem como unidades funcionais e interligados através de ações, de fluxos de informação e de transação, onde habitam as espécies digitais (DigitalEcosystem, 2007).

Os ecossistemas digitais representam, assim, em contexto educacional, sistemas de aprendizagem em rede que apoiam a cooperação, a partilha do conhecimento, o desenvolvimento de tecnologias abertas e a evolução de ambientes ricos em conhecimento, sendo que a sua criação depende exclusivamente das interações entre as espécies, as comunidades e o meio ambiente. E podem assumir qualquer tamanho, desde que comportem indivíduos da espécie humana (professores e estudantes), organismos da espécie digital (conteúdos), um ambiente digital (as tecnologias) e as interações entre os mesmos. Nestes ecossistemas digitais em rede os fatores bióticos representam as comunidades virtuais de aprendizagem, os professores, os tutores, os estudantes e os conteúdos que representam a parte viva do sistema (espécies humana e digital), enquanto as tecnologias digitais representam os fatores abióticos, as partes não vivas do ecossistema. Como num sistema biológico, os elementos da comunidade podem formar grupos espontaneamente, podendo interagir uns com os outros, sendo que para garantir o seu sucesso, cada indivíduo e cada grupo deve adaptar-se às condições ambientais e encontrar o seu "nicho" (Moreira \& Dias-Trindade, 2020).

Assim, se aceitarmos que os ecossistemas digitais de aprendizagem em rede podem representar ambientes férteis, dinâmicos, vivos e diversificados onde o conhecimento, as ideias e o espírito empreendedor podem nascer, crescer e evoluir, então é crucial reconhecer a necessidade de uma nova perspetiva na criação de cenários híbridos de aprendizagem e reconhecer a necessidade do processo ser sustentado por modelos pedagógicos colaborativos, construtivistas e de aprendizagem pela descoberta que permitam ligar vários estilos de aprendizagem.

Foi baseado nestes pressupostos que desenvolvemos a nossa investigação, procurando criar um cenário híbrido de aprendizagem, um ecossistema de educação digital e estudando o seu impacto no desenvolvimento de uma comunidade virtual.

\section{METODOLOGiA}

A disciplina "Linguagem cinematográfica e audiovisual em contexto educativo" integra o Curso de Pós-Graduação em Educação e Tecnologias da Universidade Federal de São Carlos, Brasil, sendo que a arquitetura do ecossistema digital de aprendizagem em rede foi elaborada com o recurso a sistemas de gestão de aprendizagem (LMS- Learning Management Systems); repositórios de objetos de aprendizagem; e softwares e aplicativos de colaboração e discussão da web social, como por exemplo a plataforma Moodle, o VideoAnt, plataforma de anotação de vídeo baseada na web para dispositivos móveis e desktop ou o serviço de colaboração Web Zoom Colibri. 
Entre os modelos existentes para a criação e dinamização de comunidades virtuais adotámos na nossa prática pedagógica o modelo de Community of Inquiry criado por Garrison e seus colaboradores (2000) e o modelo desenvolvido por Moreira (2017) centrado na "desconstrução" de imagens em movimento.

O primeiro modelo elaborado por Randy Garrison, Terry Anderson e Walter Archer para o ensino online designado Community of Inquiry, e posteriormente desenvolvido por Garrison e Anderson (2005), é um modelo que assenta numa perspetiva construtivista da aprendizagem e, a construção do conhecimento individual deve-se, em grande medida, ao ambiente social. Ou seja, um ambiente que favoreça uma diversidade de perspetivas pode promover a investigação, a crítica e a criatividade. Em ambiente colaborativo, o indivíduo assume o dever de dar sentido à sua experiência educativa, responsabilizando-se pelo controlo da sua aprendizagem, através da negociação de significados com o grupo (Garrison, Anderson \& Archer, 2001).

O segundo modelo baseia-se na Teoria da Flexibilidade Cognitiva, desenvolvida por Rand Spiro e colaboradores (Spiro et al., 1987) que se centra em casos que são analisados ou desconstruídos segundo múltiplas perspetivas ou temas e que considera dois processos de aprendizagem: o processo de desconstrução e o processo de travessias temáticas.

Entre as principais vantagens deste modelo, estão a aplicação de uma teoria de aprendizagem que permite uma base pedagógica consistente, o desenvolvimento da flexibilidade cognitiva dos estudantes e o estímulo da prática de análise. Este é um modelo que exige uma participação ativa na aprendizagem, começando por proporcionar uma análise em profundidade através da desconstrução do objeto audiovisual que lhe garante o aumento da flexibilidade cognitiva pelos vários exemplos que vê desconstruídos.

O ecossistema de educação digital foi estruturado com base em três unidades temáticas, desenvolvidas durante três semanas e com três momentos de avaliação. Esta avaliação traduziu-se na participação nas diferentes salas de aula virtuais criadas nos diferentes ambientes de aprendizagem do ecossistema-Moodle, VideoAnt, Zoom Colibri- e na elaboração de um trabalho final que refletiu as aprendizagens realizadas na disciplina.

A natureza da investigação levou-nos a considerar pertinente uma abordagem como a Design Based Research (DBR), de cariz qualitativo. Esta metodologia de pesquisa em educação predispõe-se a realizar investigação rigorosa para testar e aperfeiçoar ambientes de aprendizagem inovadores (Wang \& Hannafin, 2005) e caracteriza-se por "simultaneously pursues the goals of developing effective learning environments and using such environments as natural laboratories to study learning and teaching" (Sandoval \& Bell, 2004, p. 200). Trata-se de uma metodologia que se caracteriza por ultrapassar a habitual dicotomia entre investigação qualitativa e quantitativa, focando-se no desenvolvimento de pesquisas que visam a resolução de problemas significativos e práticos.

Tendo em conta o âmbito e o objetivo do estudo bem como a análise documental de alguns normativos relevantes, inspirámo-nos no Community of Inquiry Survey Instrument (CoI) desenvolvido por Garrison e colaboradores (2001), posteriormente adaptado por Moreira e Almeida (2011) à população portuguesa, para construir o nosso instrumento. 
O instrumento de avaliação levou em linha de conta as três dimensões que constituem o CoI: a Presença Cognitiva, representando a capacidade dos membros do grupo construírem significados através da comunicação dialógica, da reflexão sustentada e do discurso crítico; a Presença Social, representando a capacidade dos participantes se projetarem socialmente e emocionalmente através do meio de comunicação em uso; e a Presença Docente, representando a capacidade do docente definir a direção, o design, a facilitação da presença cognitiva e da presença social no sentido da realização dos resultados de aprendizagem. De referir que na primeira dimensão -Presença Cognitiva-, a classificação dos 20 questionários repartiu-se pelas frequências de Presença (+) e Ausência (-). Devido ao carácter avaliativo claro foram ainda sinalizadas com as expressões de Tendência Positiva (+), Tendência Negativa (-) e Hesitação/Indefinição (+/-) algumas unidades de contexto.

Tabela. Matriz para análise de conteúdo com codificação do modelo CoI

\begin{tabular}{|c|c|c|c|c|c|}
\hline \multicolumn{6}{|c|}{$\begin{array}{c}\text { Curso “Linguagem cinematográfica e audiovisual em contexto educativo” - } 2021 \\
\text { (Universidade Federal de São Carlos) }\end{array}$} \\
\hline \multicolumn{6}{|c|}{ Matriz para análise de conteúdo } \\
\hline Tema & Elementos & Categorias & Indicadores & Frequência & Tendência \\
\hline \multirow{2}{*}{$\begin{array}{l}\text { Perfil pessoal } \\
\text { do respondente }\end{array}$} & \multirow{2}{*}{ Dados pessoais } & Idade & \multirow{2}{*}{ / } & \multirow{2}{*}{ / } & \multirow{2}{*}{ / } \\
\hline & & Sexo & & & \\
\hline \multirow{10}{*}{$\begin{array}{l}\text { Construção de } \\
\text { uma } \\
\text { Comunidade } \\
\text { Virtual }\end{array}$} & \multirow{4}{*}{$\begin{array}{l}\text { Presença } \\
\text { cognitiva }\end{array}$} & $\begin{array}{c}\text { Evento } \\
\text { desencadeador }\end{array}$ & $\begin{array}{l}\text { Perceção de } \\
\text { dúvida }\end{array}$ & \multirow{4}{*}{$\begin{array}{l}\text { Presença (+) } \\
\text { Ausência (-) }\end{array}$} & \multirow{10}{*}{$\begin{array}{c}\text { Tendência Positiva } \\
(+) \\
\text { Tendência Negativa } \\
(-) \\
\text { Hesitação/ } \\
\text { Indefinição (+/-) }\end{array}$} \\
\hline & & Exploração & $\begin{array}{c}\text { Troca de } \\
\text { informação }\end{array}$ & & \\
\hline & & Integração & $\begin{array}{l}\text { Ligação de } \\
\text { ideias }\end{array}$ & & \\
\hline & & Resolução & $\begin{array}{c}\text { Troca de novas } \\
\text { ideias }\end{array}$ & & \\
\hline & \multirow{3}{*}{ Presença social } & Expressão afetiva & Emoção & & \\
\hline & & $\begin{array}{c}\text { Comunicação } \\
\text { aberta }\end{array}$ & $\begin{array}{c}\text { Livre } \\
\text { expressão }\end{array}$ & & \\
\hline & & Coesão do grupo & $\begin{array}{l}\text { Incentivo à } \\
\text { colaboração }\end{array}$ & & \\
\hline & \multirow{3}{*}{$\begin{array}{c}\text { Presença de } \\
\text { ensino/docente }\end{array}$} & $\begin{array}{c}\text { Design e } \\
\text { organização }\end{array}$ & $\begin{array}{l}\text { Definição/ } \\
\text { início dos } \\
\text { tópicos de } \\
\text { discussão }\end{array}$ & & \\
\hline & & Facilitação & $\begin{array}{l}\text { Partilha de } \\
\text { significados } \\
\text { pessoais }\end{array}$ & & \\
\hline & & Instrução direta & $\begin{array}{l}\text { Enfoque na } \\
\text { discussão }\end{array}$ & & \\
\hline
\end{tabular}

A amostra foi constituída por um grupo de vinte estudantes que responderam, no final da disciplina, a um inquérito por questionário. Para analisar os dados referentes ao estudo recorreu-se à técnica de análise de conteúdo, visto ser uma proposta que oscila entre dois polos que envolvem a investigação científica: o rigor da objetividade, da cientificidade, e a riqueza da subjetividade (Bardin, 1977). Optou-se por elencar as etapas da técnica segundo Bardin (1977), organizando a informação recolhida numa fase de pré-análise, seguida da exploração do material e do consequente tratamento dos resultados, inferência e interpretação. 
$\mathrm{Na}$ primeira fase foi realizada uma leitura flutuante, demarcaram-se os dados a analisar, formularam-se os objetivos e elaboraram-se os indicadores. Na fase de exploração do material definiu-se a codificação, a classificação e a categorização, trabalhando com unidades de registo e unidades de contexto para cada componente (assumiram-se as unidades de contexto como partes de frases escritas pelos inquiridos, ou seja, segmentos com sentido, de onde se extraíram depois as unidades de registo enquanto elementos de significação a codificar). Na última fase apostou-se no tratamento dos resultados, inferência e interpretação, com a consequente análise reflexiva e crítica. A esta análise vertical na linha de Bardin (1977) associou-se uma análise horizontal ou comparativa com recurso ao método da "análise comparativa constante" (Miles \& Huberman, 1994), com o intuito de identificar aspetos comuns e distintivos das representações e perceções dos sujeitos.

\section{RESULTADOS}

A amostra, constituída por um grupo de vinte estudantes, contou com dez participantes do sexo feminino e dez sexo masculino distribuídos por quatro faixas etárias (figura 1).

\section{Idade}

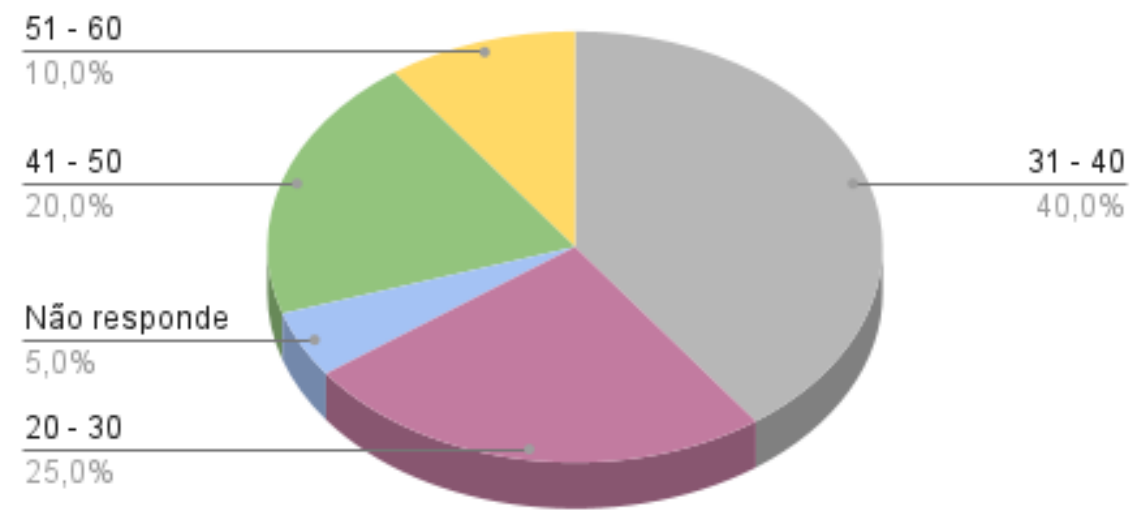

Figura 1. Idades dos participantes

Na primeira dimensão em análise, Presença Cognitiva, e como referido anteriormente, a classificação dos vinte questionários repartiu-se pelas frequências de Presença (+) e Ausência (-). A maioria (dezassete) situou-se na frequência de Presença, o que sugere que os estudantes consideram que a estratégia pedagógica desenvolvida permitiu a troca de informações e conhecimento, a ligação e a aplicação de novas ideias e a reflexão sustentada, potenciadoras do desenvolvimento de um verdadeiro pensamento crítico. À frequência de Ausência, em número muito residual (três respostas) couberam as respostas demonstrativas de uma visão relacionada com a incapacidade de o modelo promover a discussão entre estudantes, a resolução de problemas e a integração de novos conhecimentos em linha com o processo de pensamento crítico. 


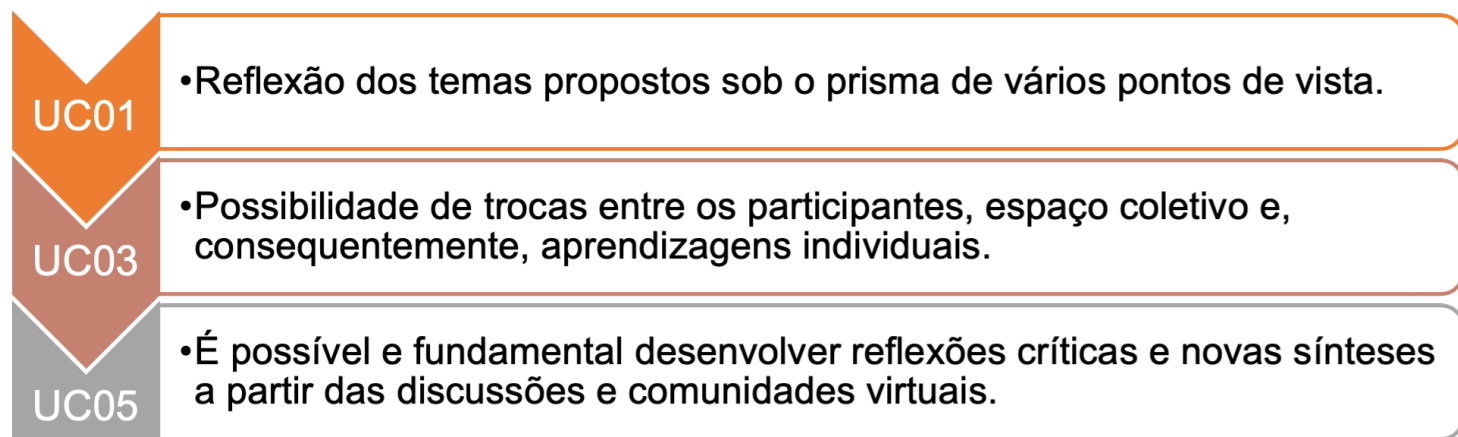

Figura 2. Exemplos de Unidades de Contexto (UC) relativas à Presença Cognitiva.

A Presença Cognitiva é a essência do quadro do CoI, no qual a construção coletiva do conhecimento ocorre. É representado pelo modelo Practical Inquiry (Garrison, Anderson \& Archer, 2001) que demonstra como a experiência interpela com a reflexão durante as fases deste componente: o evento desencadeador, exploração, integração e resolução. Tal é visível nos exemplos das Unidades de Contexto apresentadas na Figura 2 e nalgumas respostas completas dos estudantes transcritas abaixo e também nas onze Unidades de Registo (UR) categorizadas para a Presença Cognitiva (e apresentadas na Figura 3).

-ES09: “A estratégia pedagógica foi extremamente relevante para a criação de uma comunidade virtual de aprendizagem, pois foi através dela que os membros do grupo construíram significados através da comunicação dialógica";

-ES16: "Penso que houve sim uma comunicação sustentada por teorias. Muitas vezes usávamos o próprio material de apoio ou outros materiais de conhecimento dos participantes do grupo, inclusive referências diversas por sermos um grupo heterogéneo, com integrantes de várias áreas. Nota-se também nas postagens dos fóruns uma reflexão acerca da prática docente dos integrantes";

-ES18: "Foi possível e fundamental desenvolver reflexões críticas e novas sínteses a partir das discussões em comunidades virtuais".

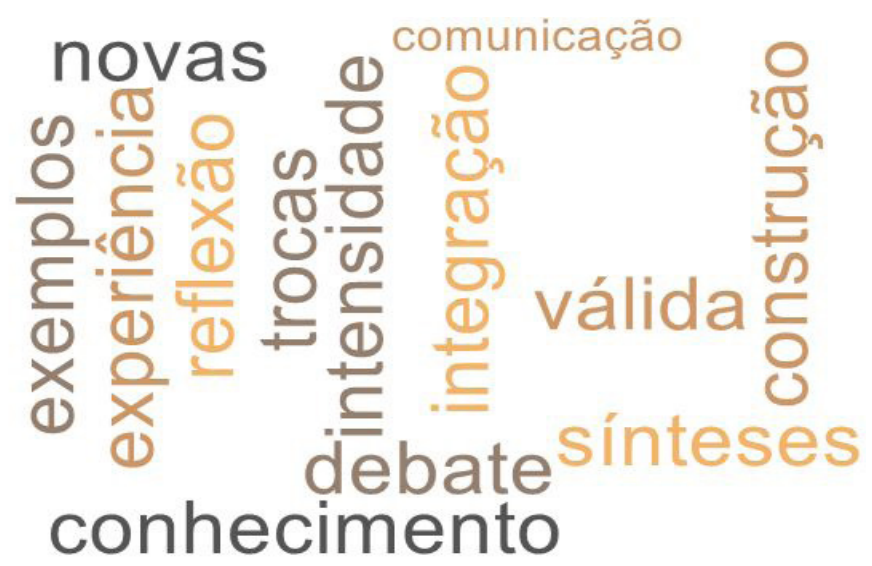

Figura 3. Unidades de Registo (UR) relativas à Presença Cognitiva. 
Os resultados relacionados com a dimensão da Presença Cognitiva revelam que os estudantes apreciaram os temas analisados e consideraram-nos como sendo relevantes para os seus interesses e práticas profissionais. As respostas obtidas sugerem também que os tópicos analisados nos fóruns despertaram curiosidade e permitiram o desenvolvimento de conhecimentos e competências (ES09). A exploração do material disponibilizado e a integração de ideias foi também potenciada por partilhas próprias de cada estudante, quer de recursos, quer de testemunhos (ES16). A fase da resolução, na forma de uma e-atividade final individual, veio comprovar o preconizado por Garrison, Anderson e Archer (2001): a fase de resolução vai para além de fóruns de discussão e, enquanto as etapas de exploração e integração são trabalhadas maioritariamente a nível reflexivo, o evento desencadeador e a resolução querem-se no mundo exterior e com dimensões práticas.

No entanto, nem todas as afirmações vão no sentido da solidez desta comunidade de aprendizagem (ES18). As respostas demonstrativas da frequência de Ausência denotam duas caraterísticas principais: uma tendência para o desinteresse em discutir, de forma crítica, em grupo e a vontade de explorar novas plataformas ou aplicações para dinamizar debates e aprendizagem colaborativa. Estes comentários, apesar de não representarem a opinião da maioria dos participantes, sugerem a necessidade de não se investir apenas numa pedagogia ou modelo pedagógico, mas considerar a pedagogia na sua dimensão plural alinhada com o uso e apropriação das tecnologias digitais em contextos híbridos e multimodais.

No que diz respeito à segunda dimensão, Presença Social, as perceções de tendência positiva representam novamente a maioria (como demonstram os exemplos de unidades de contexto da Figura 4), e demonstram que os estudantes se sentiram parte de uma comunidade de aprendizagem sólida, onde se construíram laços afetivos entre os vários elementos e onde imperaram princípios como a liberdade de expressão, a colaboração, a partilha, a autonomia e a criatividade.

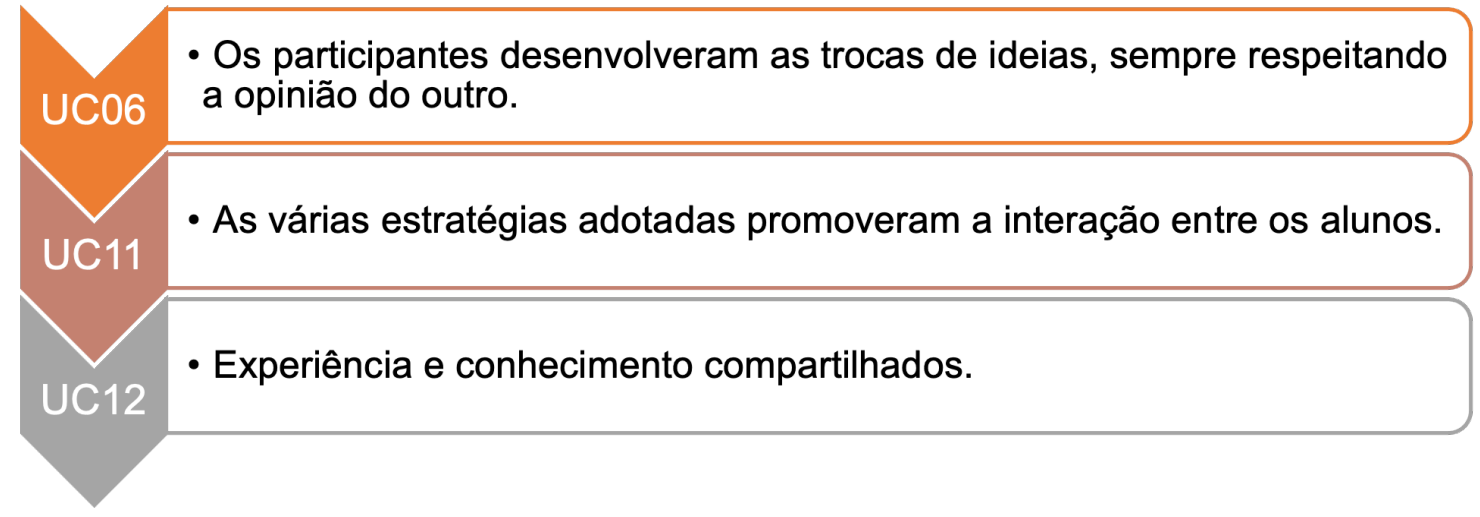

Figura 4. Exemplos de Unidades de Contexto (UC) relativas à Presença Social.

A Presença Social envolve a expressão da identidade socio-emocional de um participante no ambiente de aprendizagem. Está relacionada com a forma como os participantes do curso se identificam uns com os outros e como se podem alinhar objetivos e atividades de aprendizagem individuais e de grupo. 0 foco está na coesão e na construção de um ambiente socio-emocional onde a confiança, a abertura e a 
vontade de aprender são fatores vitais (Garrison, 2016). A maioria dos testemunhos recolhidos sublinha, pois, estas caraterísticas, reforçando a validade do modelo pedagógico e das metodologias implementadas.

-ES02: "A maioria dos participantes foi capaz de se expressar de forma aberta e expressar seus pontos de vista nas discussões das diferentes unidades";

-ES10: "As discussões em grupo ofertaram a possibilidade de reconhecimento da realidade e perceção alheia, interferindo e modificando muitas vezes o conhecimento individual e em grupo";

-ES13: “A «presença social» foi marcada pela cordialidade, pela análise construtiva do material lido e assistido e pela ampliação da perceção por meio do diálogo, das trocas e comentários dos colegas. Um ambiente que prime pela troca e diálogo é um ponto positivo na conceção da educação, em qualquer modalidade".

De destacar ainda o testemunho de um estudante - ES06 - "Acredito que essa presença social foi desenvolvida melhor em outras situações" que parece não se ter sentido satisfeito com a estratégia de implicação social. Apesar de não apresentar justificação para essa situação, tal pode estar relacionado com uma tendência também identificada por Garrison (2016) que assinala a capacidade reduzida de alguns estudantes de se identificarem com um grupo, comunicarem abertamente num ambiente de confiança e desenvolverem progressivamente as relações pessoais e afetivas através da projeção das suas personalidades individuais.

Num elemento em que imperam os indicadores relacionados com emoção, livre expressão e incentivo à colaboração, é interessante destacar as Unidades de Registo extraídas das Unidades de Contexto e perceber que elencam efetivamente essas caraterísticas.

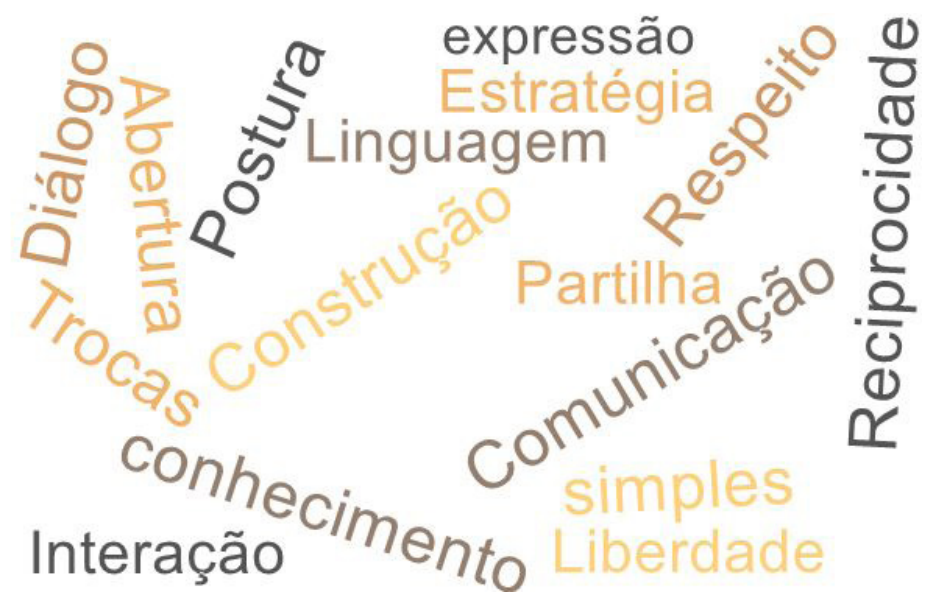

Figura 5. Unidades de Registo (UR) relativas à Presença Social.

Por fim, a terceira dimensão do modelo CoI - Presença Docente - envolve toda a conceção de uma experiência de aprendizagem, bem como a função de facilitar, moderar e mediar o processo de aprendizagem que decorre durante um curso/unidade 
curricular. A classificação das unidades de contexto e de registo mostra que a totalidade dos estudantes considerou muito relevante o papel do docente, quer na organização do ecossistema de educação digital, quer na forma como foi direcionando as discussões ou incentivando as participações, comprovando que esta é uma componente base que estabelece e sustenta a aprendizagem colaborativa e que gere o clima socio-emocional do grupo (Anderson et al., 2001).

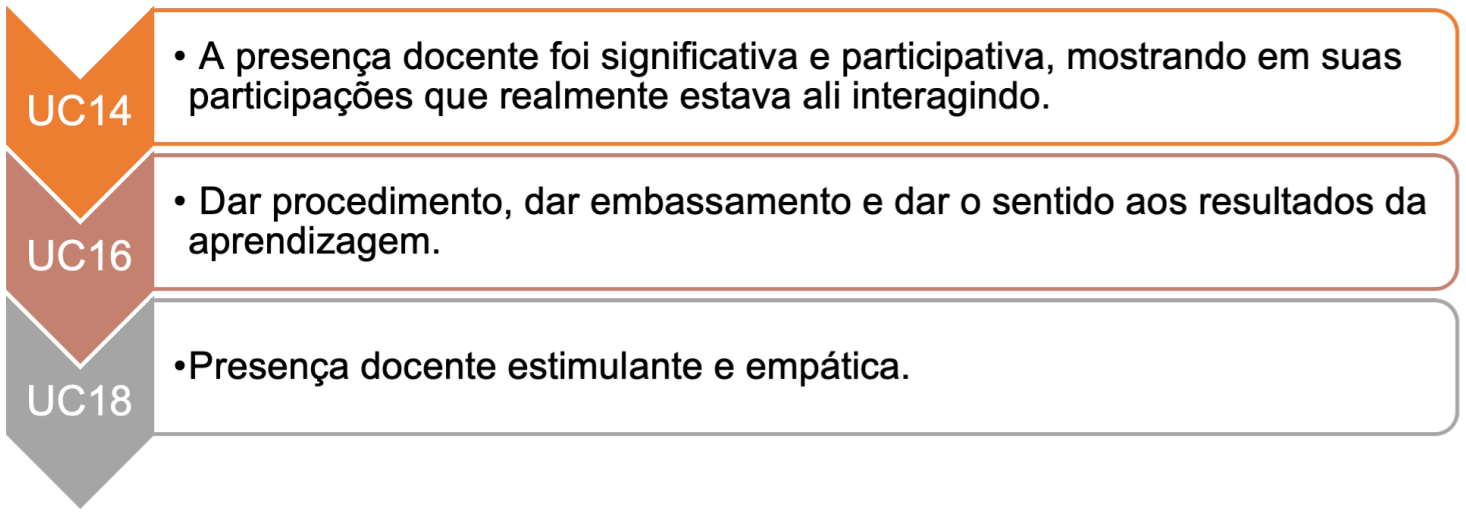

Figura 6. Exemplos de Unidades de Contexto (UC) relativas à Presença Docente.

Os testemunhos recolhidos mostram que a Presença Docente numa comunidade virtual de aprendizagem implica uma liderança forte e uma conceção distinta de todo o processo que leva ao aumento do envolvimento dos estudantes num ambiente colaborativo. Ou seja, não é uma instrução direta que estimula a aprendizagem, mas a conceção e a disponibilização de atividades de promoção de discussão e reflexão (marca identitária do modelo CoI). As respostas de todos os estudantes consubstanciam o princípio de que a qualidade do discurso e do pensamento moldados pela presença docente distingue a comunidade de aprendizagem de qualquer outra comunidade social tipicamente dominada pela interação e comunicação casuais e arbitrárias (Garrison \& Clevaland-Innes, 2005; Vaughan \& Garrison, 2019).

-ES03: "Considerei excelente a presença docente, interagindo e provocando pensamentos diferentes e reflexão, individual e coletiva, sempre no sentido de abordar o conteúdo a ser apreendido";

-ES09: "Por exemplo, se a provocação, a questão norteadora, ou mesmo as propostas de reflexão da tutora não tivessem sido feitas, não haveria a possibilidade de os diálogos construídos durante os fóruns serem tão ricos";

-ES17: "O protagonismo foi dos discentes";

-ES19: “Acredito que o trabalho dos alunos na construção de conhecimento é crucial, porém vejo que a presença do docente como orientador e facilitador é fundamental. Deste modo se deu esse componente, ou seja, tivemos liberdade para produzir conhecimento coletivamente, mas fomos orientados a todo o momento pelo professor";

-ES20: "Foi significativo e realmente importante, esta disciplina foi das melhores. 0 fato de apresentar novos olhares para o contexto educacional". 
Destacam-se ainda, nas respostas dadas, as afirmações de dois dos estudantes ES17 e ES19 - que reforçam as linhas de força e os princípios teóricos que estiveram na génese de um dos modelos pedagógicos (Moreira, 2017) utilizados nesta unidade curricular: filosofia pedagógica humanista, socioconstrutivista e colaborativa, em que os estudantes se assumem, por um lado, como indivíduos ativos, construtores do seu conhecimento, e em que os professores se assumem como moderadores que orientam a experiência educacional e que fomentam uma interação humana positiva. Este papel de professor-orientador, de professor-facilitador, de professormediador (Salmon, 2003) relaciona-se com uma nova cultura de aprendizagem intrínseco ao modelo proposto e que acaba por dar corpo a todas as unidades de registo classificadas nesta dimensão.

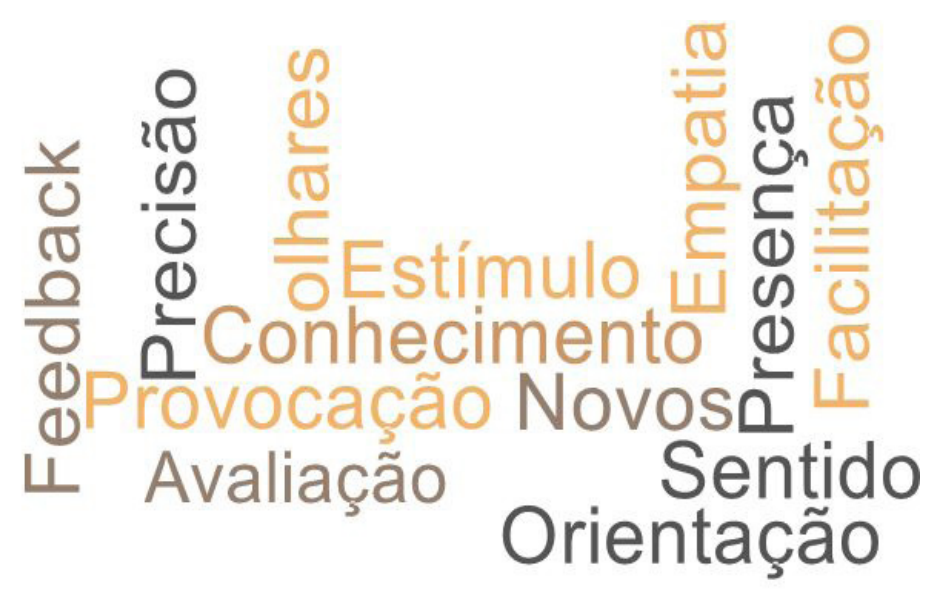

Figura 7. Unidades de Registo (UR) relativas à Presença Docente.

\section{Conclusões}

Resultados de estudos que realizámos noutros contextos (Moreira, Ferreira \& Almeida, 2013; Moreira \& Dias-Trindade, 2020; Nunes, Moreira, \& Vieira, 2020) revelam que estas abordagens pedagógicas em cenários híbridos digitais são percebidas pelos estudantes como úteis, agradáveis, flexíveis e motivadoras. A abordagem de blended (e)learning, como preferimos designar quando nos referimos a combinações eletronic, parece ser uma alternativa segura relativamente a abordagens mais clássicas que usam o espaço da geografia física como espaço nuclear e estruturante. 0 que esta investigação, também, revela é que existe "vida" para além da realidade dessa geografia física, que é possível construir cenários híbridos de aprendizagem, com uma matriz digital e virtual, promotores de uma Educação de Qualidade, tal como é preconizado nos Objetivos do Desenvolvimento Sustentável (ODS) das Nações Unidas.

Com efeito, os resultados do nosso estudo, tendo em conta as perceções dos estudantes envolvidos, revelam que, efetivamente, estes modelos pedagógicos podem ter um efeito muito positivo na criação de comunidades virtuais de aprendizagem.

Através da análise das suas perceções nas dimensões das Presenças Cognitiva e Social pudemos concluir que o ecossistema de educação digital criado nos diferentes ambientes e enriquecido com tecnologias audiovisuais permitiu criar cenários 
favoráveis para o desenvolvimento do pensamento crítico. Um ecossistema colaborativo, onde o estudante assumiu o dever de dar sentido à sua experiência educacional, através da negociação de significados com o grupo e onde se construiu conhecimento de forma coletiva, com autonomia e criatividade.

No que diz respeito à dimensão da Presença Docente concluímos que os estudantes consideram que esta foi uma dimensão muito relevante, na medida em que cabe ao professor a tarefa de implementar e desenvolver a comunidade e orientar a aprendizagem dos seus membros, criando discussões estimulantes.

Podemos, pois, afirmar, com base nas perceções destes estudantes, que a criação destes cenários de aprendizagem híbridos na realidade virtual, ancorados por modelos pedagógicos construtivistas, possuem um enorme potencial na formação e desenvolvimento de comunidades virtuais de aprendizagem.

Neste sentido, conforme temos vindo a afirmar, o sucesso da utilização destas abordagens híbridas nas realidades digital e virtual depende não só das condições tecnológicas e sociais, mas também, e fundamentalmente, das condições pedagógicas. No entanto, temos de ter consciência de que a referida renovação constante da pedagogia implica uma alteração cultural muito grande, pois obriga a repensar constantemente os papéis dos professores e dos estudantes e a relação existente entre eles. E isto não é tarefa simples. Para além disso, estes novos sistemas exigem também uma nova forma de comunicar e avaliar, onde os professores e os estudantes partilham a responsabilidade pela aprendizagem, sendo os primeiros os responsáveis pela organização inicial do ecossistema que a propicia, através do aumento da possibilidade de comunicação bidirecional, da participação ativa, da troca entre os pares, do estímulo à autonomia, mobilizando, para isso, os recursos tecnológicos disponíveis.

E os cenários híbridos de blended (e)learning, facilitando a integração e possibilitando a aproximação entre diferentes realidades, podem afirmar-se como a resposta para uma pedagogia transformadora e emancipatória.

\section{REFERÊNCIAS BIBLIOGRÁFICAS}

Anderson, T., Rourke, L., Garrison, D. R. \& Archer, W. (2001). Assessing teaching presence in a computer conferencing context. Journal of Asynchronous Learning Networks, vol. 5, núm. 2, pp. 1-17. https://doi.org/10.24059/olj. v5i2.1875

Bardin L. (1977). L'analyse de contenu. Paris: PUF.

DigitalEcosystem (2007). The Information Resource about the European approach on Digital Business Ecosystems. http://www.digital-ecosystems.org/

Garrison, D. R. (2016). Thinking collaboratively: Learning in a Community of Inquiry. New York: Routledge.

Garrison, D. \& Anderson, T. (2005). El e-learning en el siglo XXI. Investigación e práctica. Barcelona: Octaedro.

Garrison, D. R., Anderson, T. \& Archer, W. (2001). Critical thinking, cognitive presence, and computer conferencing in distance education. American Journal of Distance Education, vol. 15, núm. 1, pp. 7-23. https://doi. org/10.1080/08923640109527071 
Garrison, D. R., Anderson, T. \& Archer, W. (2000). Critical inquiry in a text-based environment: computer conferencing in higher education. The Internet and Higher Education, vol. 2, núm. 2-3, pp. 87-105. https://doi.org/10.1016/ S1096-7516(00)00016-6

Garrison, D. R. \& Cleveland-Innes, M. (2005). Facilitating cognitive presence in online learning: Interaction is not enough. American Journal of Distance Education, vol. 19, núm. 3, pp. 133-148. https://doi.org/10.1207/s15389286ajde1903_2

Harasim. L. (2015). Educação online e as implicações da inteligência artificial. Revista da FAAEBA, Educação e Contemporaneidade, vol. 24, núm. 44, pp. 2539. https://www.revistas.uneb.br/index.php/faeeba/article/view/12091

Miles, M. \& Huberman, M. (1994). Drawing valid meaning from qualitative data: toward a shared craft. Educational Researcher, vol. 13, pp. 20-30.

Monteiro, A., Moreira, J. A. \& Lencastre, J. A. (2015). Blended (E)learning na sociedade digital. Whitebooks: Santo Tirso.

Moreira, J. A. (2017). A pedagogical model to deconstruct moving pictures in virtual learning environments and its impact on the self-concept of postgraduate students. Journal of e-Learning and Knowledge Society, vol. 13, núm. 1, pp. 77-90. https://doi.org/10.20368/1971-8829/1255

Moreira, J. A., Ferreira, A. G. \& Almeida, A. C. (2013). Comparing communities of inquiry in higher education students: one for all or one for each? OpenPraxis, vol. 5, núm. 2, pp. 165-178.

Moreira, J. A. \& Almeida, A. C. (2011). How reliable and consistent is our learning community of inquiry? Psychometric qualities of the community of inquiry survey instrument applied to a sample of higher education Portuguese students. EduLearn 2011. In International Conference on Education and New Learning Technologies, 4th-6th July, Barcelona (Spain).

Moreira J. A. \& Dias-Trindade, S. (2020). Online learning environments enriched with audiovisual technologies and its impact on the construction of virtual communities in Higher Education in prison context. Journal of e-Learning and Knowledge Society, vol. 16, núm. 1, pp. 9-16. https://doi.org/10.20368/19718829/113503

Moreira, J. A. \& Ferreira, A. G. (2021). Blended and hybrid environments in Higher Education in times of the pandemic. In S. Gonçalves \& S. Majhanovich (coord.). Pandemic and remote teaching in Higher Education (pp. 9-20). Coimbra: CINEP, Portugal.

Nunes, C., Moreira, J. A. \& Vieira, C. (2020). Comunidades virtuais de aprendizagem e competências-chave para o século XXI no ensino superior a distância. Revista Educaonline, vol. 14, núm. 2, pp. 1-22. http://hdl.handle. net/10400.2/10413

Salmon, G. (2003). E-ativities: The key to teaching and learning online. Londres: Routledge.

Sandoval, W. \& Bell, P. (2004) Design-based research methods for studying learning in context: Introduction. Educational Psychologist, vol. 39, núm. 4, pp. 199-201. https://doi.org/10.1207/s15326985ep3904_1

Spiro, R., Vispoel, W., Schmitz, J., Samarapungavan, A. \& Boerger, A. (1987). Knowledge aquisition for application: Cognitive flexibility and transfer in complex content domains. In B. Britton \& C. Glynn C. (eds.). Executive control in processes in reading (pp. 177-199). New Jersey: Lawrence Erlbaum Associates. 
Vaughan, N. \& Garrison, R. (2019). How blended learning can support a faculty development community of inquiry. Online Learning, vol. 10, núm. 4, pp. http://dx.doi.org/10.24059/olj.v10i4.1750

Wang, F. \& Hannafin, M. J. (2005). Design-based research and technology-enhanced learning environments. Educational Technology Research and Development, vol. 53, núm. 4, pp. 5-23. 